\title{
DETECTING LINEAR FEATURES BY SPATIAL POINT PROCESSES
}

\author{
Dengfeng Chai ${ }^{\mathrm{a}}$, Alena Schmidt ${ }^{\mathrm{b}}$, Christian Heipke $^{\mathrm{b}}$ \\ ${ }^{a}$ Institute of Spatial Information Technique, Zhejiang University, China - \\ chaidf@zju.edu.cn \\ ${ }^{\mathrm{b}}$ Institute of Photogrammetry and GeoInformation, Leibniz Universität Hannover, Germany - \\ alena.schmidt@ipi.uni-hannover.de, heipke@ipi.uni-hannover.de
}

\section{ICWG III/VII}

KEY WORDS: Linear Feature, Feature Detection, Spatial Point Processes, Global Optimization, Simulated Annealing, Markov Chain Monte Carlo

\begin{abstract}
:
This paper proposes a novel approach for linear feature detection. The contribution is twofold: a novel model for spatial point processes and a new method for linear feature detection. It describes a linear feature as a string of points, represents all features in an image as a configuration of a spatial point process, and formulates feature detection as finding the optimal configuration of a spatial point process. Further, a prior term is proposed to favor straight linear configurations, and a data term is constructed to superpose the points on linear features. The proposed approach extracts straight linear features in a global framework. The paper reports ongoing work. As demonstrated in preliminary experiments, globally optimal linear features can be detected.
\end{abstract}

\section{INTRODUCTION}

Image features play a fundamental role in many tasks of image analysis. For example, both surface reconstruction and object extraction usually rely on some distinguishable features. Feature detection has been investigated widely in the communities of photogrammetry and remote sensing, computer vision and pattern recognition. However, few works were dedicated to modeling feature shape in a global framework. This paper address this issue in the context of detecting linear features. Linear features offer salient cues for region boundaries and object contours.

\subsection{Related Work}

Image features refer to image primitives such as points, lines, curves and regions. Corner detectors and interest point detectors have been developed to detect point features (Förstner and Gülch, 1987, Harris and Stephens, 1988, Tomasi and Kanade, 1991, Shi and Tomasi, 1994, Smith and Brady, 1997, Lowe, 2004, Rosten and Drummond, 2006, Bay et al., 2008, Rublee et al., 2011). Region detectors have been proposed to detect region features (Kadir et al., 2004, Tuytelaars and Van Gool, 2004, Leutenegger et al., 2011, Alahi et al., 2012). Both straight lines and curves are linear features. Their detection is formulated as edge detection, line detection, boundary detection or contour detection.

Edge detection aims at detecting edges, which refer to abrupt changes of brightness. Basically, sharp changes are computed by differential operations, which is achieved by differentiation based filters such as Sobel, Prewitt, Roberts, Laplacian of Gaussian, etc. These filters are simple and sensitive to noise. This disadvantage is significantly overcome by the Canny edge detector (Canny, 1986). Its success depends on the definition of three comprehensive criteria, namely good detection, good localization and single-pixel response, for the computation of edges. Edge detection is formulated as an optimization with respect to these criteria. Benefiting from its solid mathematical foundation, this detector has proven to be reliable and has gained popularity since it was first published. Besides, there are some extended and adapted versions (Deriche, 1987, McIlhagga, 2011, Xu et al.,
2014). However, feature shape is not modeled in the computational framework.

Lines are pairs of anti-parallel edges. Depending on the width of the linear feature to be detected and the contrast with respect to the neighborhood, line detection can be advantageous to edge detection. The most prominent example of line detection is the Steger operator (Steger, 1998).

Boundary detection refers to detecting boundaries between different regions. Boundaries are indicated by edges, excluding those inside a region. However, there are typically a lot of edges inside a textured region. This issue is addressed by the $\mathrm{Pb}$ (probabilityof-boundary) edge detection algorithm (Martin et al., 2004). For each pixel it fuses brightness gradient, texture gradient and color gradient to calculate the probability of being a boundary pixel. Further, MS- $\mathrm{Pb}$ (Multiple Scale $\mathrm{Pb}$ ) integrates $\mathrm{Pb}$ in multiple scales (Ren, 2008), and gPb (global Pb) introduces global information into the MS- $\mathrm{Pb}$ (Arbelaez et al., 2011). The gPb detector can detect boundaries between texture regions. However, none of them models boundary shape.

Contour detection is dedicated to extracting outlines of objects of interest. Some approaches group edges, lines or boundary fragments into contours (Zhu et al., 2007, Arbelaez et al., 2011, Ming et al., 2013, Payet and Todorovic, 2013). The other approaches fit active contour models to images (Kass et al., 1988, Mishra et al., 2011, Dubrovina et al., 2015). Although shape is modeled and utilized by these approaches, modeling is performed not in a global framework.

Spatial point processes are stochastic models for spatial entities. The relationships among entities can be considered by a prior model, and, the shapes of the entities can be represented by some geometric marks. For example, linear features and line-networks are represented by line segments and polylines (Stoica et al., 2004, Lacoste et al., 2005, Lacoste et al., 2010, Chai et al., 2013, Schmidt et al., 2015), building outlines are represented by rectangles (Ortner et al., 2007, Ortner et al., 2008), and tree crowns are represented by circles and ellipses (Perrin et al., 2005). But these 


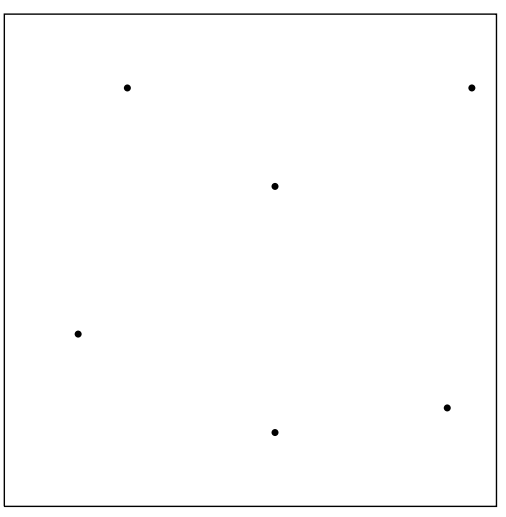

(a) Spatial Point Process

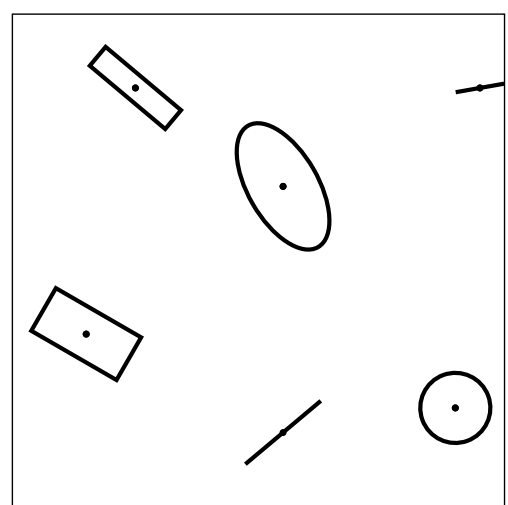

(b) Marked Point Process

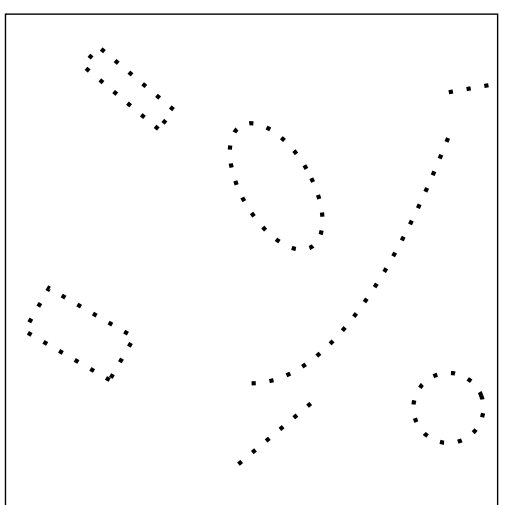

(c) Linear Point Process

Figure 1: Overview of three kinds of spatial point processes.

marks are too specific to represent general linear shapes. Although a combination of above marks integrates their capacities (Lafarge et al., 2010), they are not general enough to represent freeform linear features. Alternatively, stochastic models can be employed to segment an image into regions, whose boundaries are freeform linear features ( $\mathrm{Tu}$ and $\mathrm{Zhu}, 2002)$. However, regions instead of boundaries are modeled explicitly.

\subsection{Motivation and Contribution}

Most feature detectors are not developed in a global framework. Also, they do not deal with feature shape and uncertainty. While marked point processes do provide a global model for feature shape and uncertainty, in existing approaches linear features are represented by geometric marks. Apart from losing generality, the space of solutions is expanded significantly since additional parameters are involved in decribing the geometric marks. These additional parameters impair both detection quality and efficiency.

With reference to Fig. 1 a point is an atomic element in mathematics, and a string of points forms a (possibly straight) curve. In turn, a set of connected curves encloses a region. Motivated by this common sense observation, in this paper a linear feature is represented by a string of points. Further, the paper proposes a spatial point process, in which points are inclined to form curves as shown in Fig. 1(c). The paper makes several contributions:

- Spatial point process: Based on the proposed model for a spatial point process, points can form linear shapes. This characteristic assures its potential in modeling and analyzing linear shapes.

- Feature representation: The proposed model is general enough to represent freeform linear features. Edges, lines, boundaries and contours can all be represented in this model.

- Global feature detection: Linear feature detection is formulated as a global optimization problem. The globally optimal solution can be reached very efficiently since no extra parameters are involved in the computation.

The rest of the paper is organized as follows. First, some background information on spatial point processes is introduced in Sec. 2 Second, the novel approach for linear feature extraction is proposed in Sec. 3 Then, experimental results are presented in Sec. 4 Finally, conclusions are drawn in Sec. 5

\section{FEATURE EXTRACTION BASED ON MARKED POINT PROCESSES}

\subsection{Spatial Point Processes}

A spatial point process is a useful model for a random pattern of points as depicted in Fig. 1(a). For a $k$-dimensional space $R^{k}$, let $F \subset R^{k}$ be a compact set, let $\omega=\left\{\omega_{1}, \ldots, \omega_{n}\right\}$ be a configuration having $n$ unordered points $\omega_{i} \in F$, let $\Omega_{n}$ be the set of configurations that consist of $n$ points. A point process on $F$ is a mapping $\Psi$ from a probability space to the set of configurations $\Omega=\bigcup_{n=1}^{\infty} \Omega_{n}$, such that, for all bounded Borel sets $S \subset F$, the number of points $N_{\Psi}(S)$ falling in $S$ is a finite random variable.

A Poisson process in the plane with uniform intensity measure $\nu\left(\right.$.) is a point process in $F \in R^{2}$, such that, for every bounded closed set $S \in F$, the number of points falling in this region follows a Poisson distribution with mean $\nu(S)$, and the number of points are independent for disjoint regions.

Complex point processes are specified by a probability density $h($.$) defined on \Omega$ and a reference measure $\mu($.$) under the condi-$ tion that the normalization constant of $h($.$) is finite:$

$$
\int_{\omega \in \Omega} h(\omega) d \mu(\omega)<\infty
$$

The measure $\mu($.$) having the density h($.$) is usually defined via$ the intensity measure $\nu($.$) of a homogeneous Poisson process.$ For a more detailed definition of point processes the reader is referred to (Møller and Waagepetersen, 2004).

\subsection{Feature Extraction Based on Marked Point Processes}

To extract features from images, a data term measuring the consistency between the points and the image is introduced into the density $h($.$) , and a prior term reflecting the spatial interactions$ among the points is also introduced into the density. This density can be expressed as a product of a data term $h_{d}($.$) and a prior$ term $h_{p}($.$) :$

$$
h(.)=h_{p}(.) h_{d}(.)
$$

Feature extraction is achieved by searching for the configuration $\omega^{\star}$ maximizing the probability density as

$$
\omega^{\star}=\underset{\omega \in \Omega}{\arg \max } h(\omega) .
$$


Existing approaches typically employ geometric marks such as line segments to represent shapes. As depicted in Fig. 1(b), the geometric marks are very specific, they cannot represent general shapes. Furthermore, the configuration space $\Omega$ is expanded dramatically by the extra parameters for the marks. Both, detection quality and efficiency are impaired within this complex space.

\section{LINEAR FEATURE EXTRACTION BASED ON SPATIAL POINT PROCESSES}

\subsection{Linear Feature Representation}

This paper represents a linear feature by a string of points instead of a geometric mark. As shown in Fig. 1(c), a line segment is represented by a set of points sampled on the segment. Any other linear features such as freeform curves are also represented by their sample points.

The suggested model contains a data term measuring the consistency between the points and the image data. Further, this paper proposes a prior model for spatial point processes such that linear configurations become more probable than non-linear configurations and straight ones are prefered to curved ones.

\subsection{A Prior Model for Linear Configurations}

A prior probability of a configuration $\omega$ is measured by three different criteria: the sparsity of its points, the neighborhood of its points and the curvature of the linear configuration. The density $h_{p}$ is a product of three densities:

$$
h_{p}(\omega) \propto h_{s}(\omega) h_{n b}(\omega) h_{c}(\omega)
$$

where $h_{s}(\omega), h_{n b}(\omega)$ and $h_{c}(\omega)$ are three densities based on the criteria of sparsity, neighborhood and curvature, respectively.

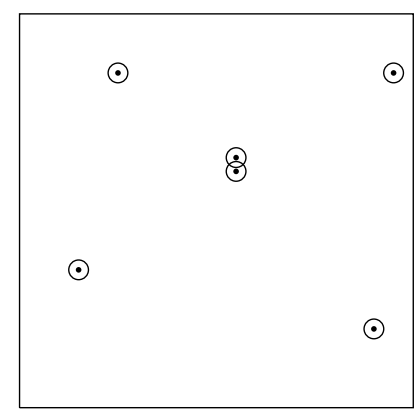

Figure 2: Sparsity is assured by penalizing closely neighboring points indicated by the overlapping disks.

Sparsity: Configurations of sparse points are assured by penalizing small distances between neighboring points. Let a disk of radius $r_{1}$ be the influence area of each point as illustrated in Fig. 2. When two points are closer than $2 r_{1}$, their disks overlap. To avoid overlapping, the distance between any two points must be larger than $2 r_{1}$.

The density for sparsity is defined as

$$
h_{s}(\omega) \propto \alpha^{s\left(\omega, r_{1}\right)},
$$

where $\alpha$ is a parameter of this model, $s\left(\omega, r_{1}\right)$ is calculated as follows:

$$
s\left(\omega, r_{1}\right)=\sum_{\omega_{i}, \omega_{j} \in \omega} 1\left[d\left(\omega_{i}, \omega_{j}\right) \leq 2 r_{1}\right]
$$

where $d\left(\omega_{i}, \omega_{j}\right)$ is the distance between $\omega_{i}$ and $\omega_{j}$.

The above model penalizes small distances between neighboring points. For $0<\alpha<1$, it is a soft constraint, points are allowed to be closer than $2 r_{1}$, but they are less probable.

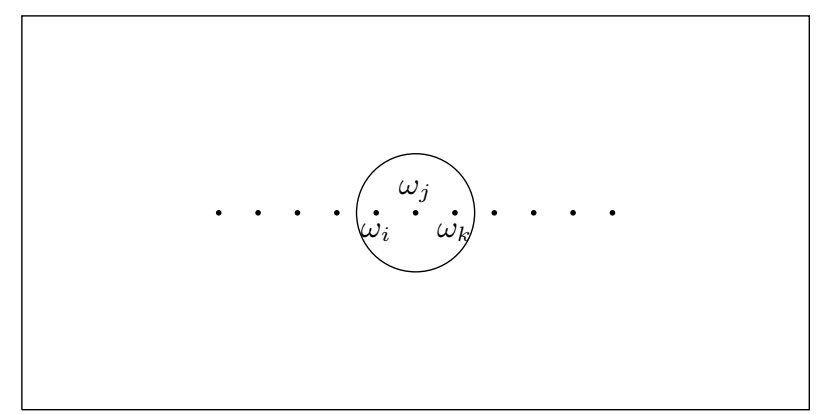

Figure 3: Each point on a line excluding the first and last point has two neighbors within a disk, i.e., a preceding point and a succeeding point.

Neighborhood: As one tracks points along a line, each point has a preceding point and a succeeding point, excluding the first point and the last point. As depicted in Fig. 3, point $\omega_{j}$ has a preceding point $\omega_{i}$ and a succeeding point $\omega_{k}$. This is a basic characteristic of linear features; it is introduced into the neighborhood density as follows

$$
h_{n b}(\omega)=\beta^{n b\left(\omega, r_{2}\right)},
$$

where $\beta$ is a parameter of this model, and $n b\left(\omega, r_{2}\right)$ is calculated as

$$
n b\left(\omega, r_{2}\right)=\sum_{\omega_{j} \in \omega}\left|\operatorname{deg}\left(\omega_{j}, r_{2}\right)-2\right|
$$

where $\operatorname{deg}\left(\omega_{j}, r_{2}\right)$ is the degree of $\omega_{j}$, i.e. the number of points within a disk of radius $r_{2}$ around $\omega_{j}$. Note that typically $r_{2}$ is assumed to be larger than $r_{1}$.

$$
\operatorname{deg}\left(\omega_{j}, r_{2}\right)=\sum_{\omega_{i} \in \omega, \omega_{i} \neq \omega_{j}} 1\left[d\left(\omega_{i}, \omega_{j}\right) \leq r_{2}\right]
$$

where $d\left(\omega_{i}, \omega_{j}\right)$ is the distance between $\omega_{i}$ and $\omega_{j}$.

For $0<\beta<1$, points with two neighbors within a disk of radius $r_{2}$ are preferred and the other points are penalized.

Curvature: The local curvature at a point of a linear feature is described by the angle $\delta$ defined by its preceding and succeeding point as illustrated in Fig. 4. Note that the curvature term is only applied for points with exactly two neighbors, as otherwise its computation in the described way is either not possible (one neighbor) or ambiguous (three or more neighbors).

In our approach linear features are assumed to have small curvatures; this is why we say we prefer straight linear features. In 


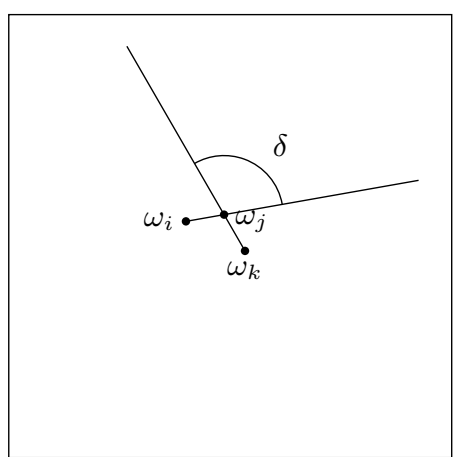

Figure 4: The angle defined by three successive points.

turn, small curvature is indicated by an angle close to $\pi$. This angle is introduced into the curvature density as follows

$$
h_{c}(\omega)=\gamma^{c(\omega)},
$$

where $\gamma$ is a parameter of the model, and $c(\omega)$ is calculated by

$$
\begin{gathered}
c(\omega)=\sum_{\omega_{j} \in \omega} \operatorname{ang}\left(\omega_{j}\right), \\
\operatorname{ang}\left(\omega_{j}\right)=\left\{\begin{array}{ll}
0 & \operatorname{deg}\left(\omega_{j}\right) \neq 2 \\
1-\cos \delta & \operatorname{deg}\left(\omega_{j}\right)=2
\end{array},\right.
\end{gathered}
$$

where $\omega_{i}$ and $\omega_{k}$ are two neighbors of $\omega_{j}$.

For $0<\gamma<1$, points with large curvature are penalized.

\subsection{Data Consistency of Linear Features}

Assuming the data for the points to be independent, the data density can be written as a product of local terms

$$
h_{d}(\omega) \propto \prod_{i=1,2, \ldots n} h_{d}\left(\omega_{i}\right)
$$

where the local term $h_{d}\left(\omega_{i}\right)$ for a point $\omega_{i}$ measures its consistency with a linear feature, i.e. the likelihood of being either a corner or an edge. The local term is computed by

$$
h_{d}\left(\omega_{i}\right)=\exp \left(\lambda_{1}+\lambda_{2}\right)
$$

where $\lambda_{1}$ and $\lambda_{1}$ are two eigenvalues of the Harris matrix, which is calculated as the covariance matrix $M$ of the derivatives of the image $I$ with respect to the pixel neighborhood

$$
M=\left(\begin{array}{cc}
\sum_{N\left(\omega_{i}\right)} I_{x}^{2} & \sum_{N\left(\omega_{i}\right)} I_{x} I_{y} \\
\sum_{N\left(\omega_{i}\right)} I_{x} I_{y} & \sum_{N\left(\omega_{i}\right)} I_{y}^{2}
\end{array}\right),
$$

where $I_{x}=\frac{\partial I}{\partial x}$ and $I_{y}=\frac{\partial I}{\partial y}$ are two derivatives, and $N\left(\omega_{i}\right)$ is a window centered at $\omega_{i}$.

As pointed out in (Harris and Stephens, 1988), $\lambda_{1}+\lambda_{2}=\operatorname{Tr}(M)$ measuring the flatness at the point. Large values indicate either a corner or an edge. Therefore, this data term can be used to find points at object boundaries.

\subsection{Linear Feature Extraction}

Linear feature extraction is formulated as finding the optimal configuration of a spatial point process. The optimal configuration $\omega^{\star}$ maximizes the probability density

$$
\omega^{\star}=\underset{\omega \in \Omega}{\arg \max } h_{s}(\omega) h_{n b}(\omega) h_{c}(\omega) h_{d}(\omega)
$$

This is not a conventional optimization problem since the configuration space has variable dimensions and the probability density is multi-modal. Simulated annealing is employed to successively simulate a series of probability distributions which converges to a distribution concentrated on the optimal configuration (Kirkpatrick et al., 1983). The Reversible Jump Markov Chain Monte Carlo (RJMCMC) sampler is embedded into the optimization procedure to take into account the variable dimension of the configuration space and to simulate the expected probability distribution (Green, 1995).

3.4.1 Searching the Optimal Configuration: Consider a spatial point process, in which each configuration $\omega$ has the probability density

$$
h_{T}(\omega)=[h(\omega)]^{1 / T}=\left[h_{s}(\omega) h_{n b}(\omega) h_{c}(\omega) h_{d}(\omega)\right]^{1 / T}
$$

where $T>0$ is a temperature parameter. When $T \rightarrow \infty, h_{T}(\omega)$ defines a uniform distribution on $\Omega$; for $T=1, h_{T}(\omega)=h(\omega)$; and as $T \rightarrow 0, h_{T}(\omega)$ concentrates on the optimal configuration $\omega^{\star}$. When the temperature starts from a large value and decreases according to a logarithmic function, it is guaranteed that the globally optimal configuration is found as the temperature approaches zero. In practice, a faster geometric decrease gives an approximate solution, in general close to the optimum (Baddeley and Lieshout, 1993).

The RJMCMC sampler is adopted to simulate a discrete Markov Chain $\left(X_{t}\right)_{t \in \mathbb{N}}$, which converges to $h_{T}(\omega)$. At each transition, one point of the current configuration $\omega$ is perturbed to generate a new configuration $\omega^{\prime}$ according to a kernel $Q(\omega \rightarrow$.). The configuration $\omega^{\prime}$ is then accepted as the new state of the chain with a probability $\min (1, R)$, in which the Green ratio is calculated as

$$
R=\frac{Q\left(\omega^{\prime} \rightarrow \omega\right)}{Q\left(\omega \rightarrow \omega^{\prime}\right)}\left(\frac{h\left(\omega^{\prime}\right)}{h(\omega)}\right)^{\frac{1}{T}}
$$

The kernel $Q$ can be a mixture of some sub-kernels $Q_{m}$

$$
Q(\omega \rightarrow .)=\sum_{m} p_{m} Q_{m}(\omega \rightarrow .)
$$

where $p_{m}$ is the probability of choosing the sub-kernel $Q_{m}$. The kernel mixture must allow any configuration in $\Omega$ to be reached from any other configuration in a finite number of perturbations (irreducibility condition of the Markov chain), and each sub-kernel has to be reversible, i.e. able to propose the inverse perturbation.

3.4.2 Transition Kernels: The birth and death kernel $Q_{B D}$ and the translation kernel $Q_{T}$ are developed in the sampler. These two kernels guarantee the irreducibility condition of the Markov chain. 
Birth and death kernel: The uniform birth and death kernel inserts a point into the current configuration $\omega$ and deletes a point from the current configuration $\omega$, respectively. Adding and removing a point corresponds to jumps into a higher dimensional and a lower dimensional subspace, respectively. The green ratio for a birth kernel is given by

$$
R=\frac{p_{d} \lambda_{F}}{p_{b} n\left(\omega^{\prime}\right)}\left(\frac{h_{s}\left(\omega^{\prime}\right) h_{n b}\left(\omega^{\prime}\right) h_{c}\left(\omega^{\prime}\right) h_{d}\left(\omega_{i}^{\prime}\right)}{h_{s}(\omega) h_{n b}(\omega) h_{c}(\omega)}\right)^{\frac{1}{T}}
$$

where $\lambda_{F}$ is the Poisson parameter representing the expected number of points in the domain $F$ (the whole image), $n\left(\omega^{\prime}\right)$ is the number of points in the proposed configuration $\omega^{\prime}, \omega_{i}^{\prime}$ is the inserted point, and $p_{d}$ (resp. $p_{b}$ ) is the probability of choosing a death (resp. a birth) kernel. In our experiments we set $p_{d}=p_{b}=0.5$. In case of a death, the proposition kernel ratio corresponds to the inverse of the birth's ratio.

Translation kernel: The translation kernel moves a point $\omega_{i}$ to a new point $\omega_{i}^{\prime}$. The green ratio for a translation kernel is given by

$$
R=\left(\frac{h_{s}\left(\omega^{\prime}\right) h_{n b}\left(\omega^{\prime}\right) h_{c}\left(\omega^{\prime}\right) h_{d}\left(\omega_{i}^{\prime}\right)}{h_{s}(\omega) h_{n b}(\omega) h_{c}(\omega) h_{d}\left(\omega_{i}\right)}\right)^{\frac{1}{T}}
$$

In our experiments, a point is moved in a square centered around its old position and the length of each side is set to be $r_{1}$.

3.4.3 Sampling Driven by Gradients: The above scheme samples the configuration space uniformly. It spends a lot of time checking impossible configurations. The points are usually expected to lie on edges with large gradients. Motivated by the Data Driven Markov Chain Monte Carlo (DDMCMC) approach ( Tu and Zhu, 2002), a scheme utilizing gradient information is developed to improve the sampling.

First, the point must be sampled at the pixel locations, i.e. the sample points stem from the set of pixels:

$$
\omega_{i} \in P
$$

where $P$ is the set of pixels.

Then, each pixel has a weight proportional to its gradient magnitude

$$
w(p) \propto \sqrt{I_{x}^{2}+I_{y}^{2}},
$$

where $I_{x}=\frac{\partial I}{\partial x}$ and $I_{y}=\frac{\partial I}{\partial y}$ are the two derivatives. The weights of all pixels are normalized such that its sum equals one.

Finally, uniform birth and death is replaced with gradient driven birth and death. In uniform birth and death, each pixel has the same chance of being selected as a new point, while in gradient driven birth and death the pixels having larger gradient have more chance of being selected as a new point. The chance is proportional to its gradient.

\section{EXPERIMENTAL RESULTS}

The described method was implemented and some preliminary tests were conducted to obtain a first insight into the behavior of the suggested approach. The experiments consist of model simulation and feature detection, the results are reported in the following. The parameters selected for the experiments are presented in Tab. 1 .

\begin{tabular}{|c|c|c|c|c|c|}
\hline$\lambda$ & $\alpha$ & $\beta$ & $\gamma$ & $r_{1}$ (pixels) & $r_{2}$ (pixels) \\
\hline 200 & 0.9 & 0.9 & 0.9 & 5 & 10 \\
\hline \multicolumn{1}{|c|}{ Table 1: rarameters for experiments }
\end{tabular}

Fig. 5 presents two simulations based on two models. An optimal configuration based on the homogenous Poisson point process is presented in the left figure. The points are totally random and no linear features appear. In contrast, the right figure presents an optimal configuration based on the proposed model. The points are inclined to form lines and curves as expected.

Fig. 6 presents the results of feature detection. The left column presents the original images. The two middle columns depict features detected by the Harris detector (Harris and Stephens, 1988) and the Canny detector (Canny, 1986). The right column depicts features detected by the proposed approach. The detected features are represented by the shown points. The detected points representing the linear features are well placed on the region boundaries and they clearly illustrate the outline of the objects. Moreover, they are general enough to represent any freeform features and objects.

The data term is calculated once for each pixel and is stored as an image for use in the sampling procedure. In this way calculation efforts are minimized and a better efficiency is achieved.

\section{CONCLUSION}

This paper proposes a novel approach for linear feature detection. Feature detection is formulated as finding an optimal configuration of a spatial point process. Based on this formulation, a prior model is proposed to favor straight linear configurations, and a data model is constructed to draw the points towards linear features. The proposed approach detects linear features in a globally optimal framework. As demonstrated by the experiments, features can in principle be detected and they sit on the boundaries with good accuracy.

One limitation of the approach is that linear features are not detected explicitly. The model can only serve as an intermediate representation between edges and contours, which are lowlevel and high-level features, respectively. Converting the detected points into contours is worthy to investigate in the future. Further, the model does not include any topological constraints. Therefore, a network containing junctions cannot be properly extracted. Representing junctions without marks is also an issue which needs further attention. Finally, the data term can be improved by introducing a more sophisticate response, e.g. by introducing the concep of probability-of-boundary (Martin et al., 2004).

\section{ACKNOWLEDGEMENTS}

The work is supported by the National Natural Science Foundation of China (No.41071263, 41571335) and Zhejiang Provincial Natural Science Foundation of China (No.LY13D010003). It is also supported by Key Laboratory for National Geographic Census and MonitoringNational Administration of Surveying, Mapping and Geoinformation (No. 2014NGCM). 


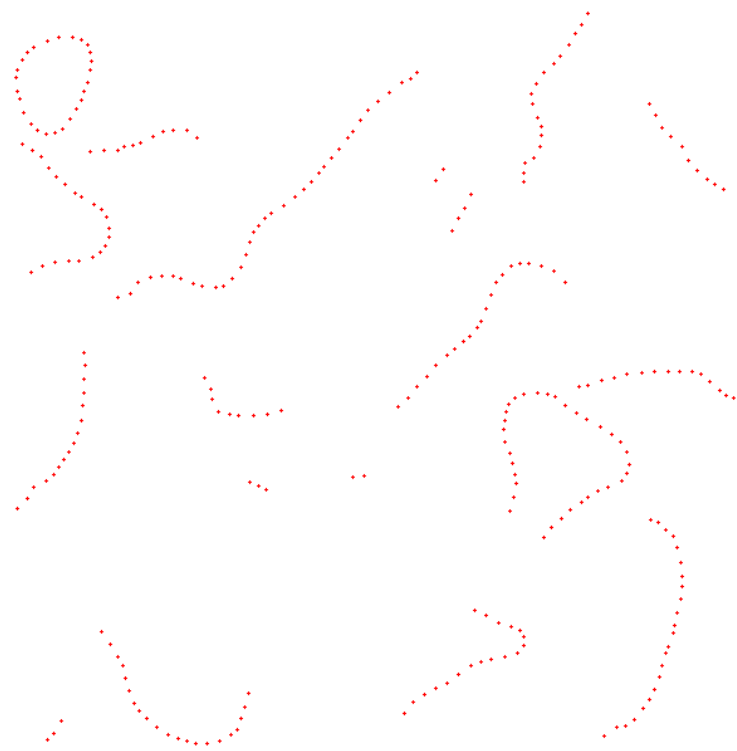

(b) Linear

Figure 5: Two simulation results: on the left an optimal configuration of a homogenous Poisson point process is shown, in which points are distributed randomly. To the right a configuration with density defined by the proposed model is illustrated, in which points are distributed along linear curves.

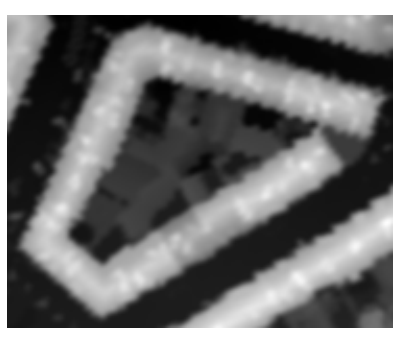

(a) Image

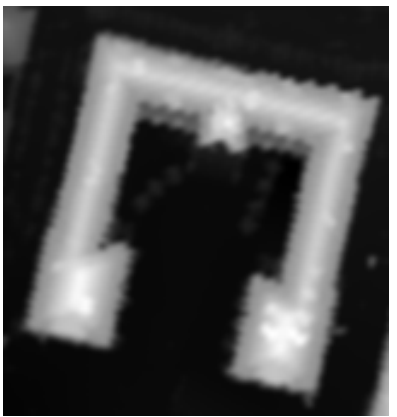

(e) Image

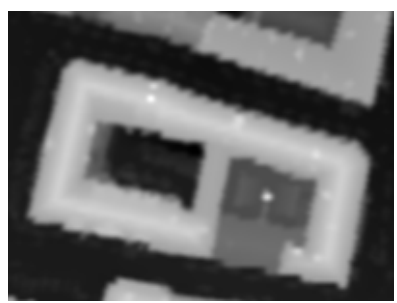

(i) Image

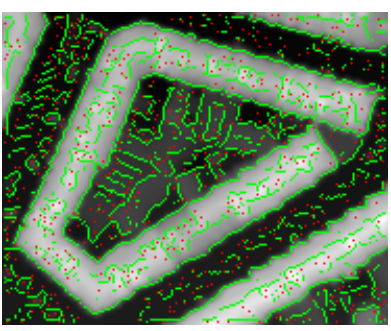

(b) Harris

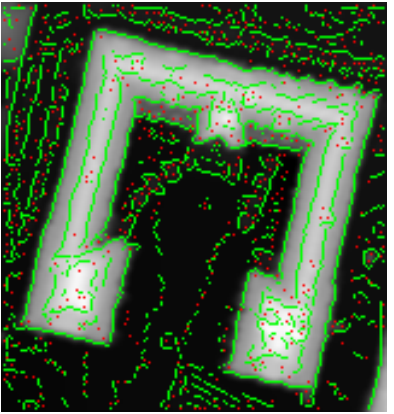

(f) Harris

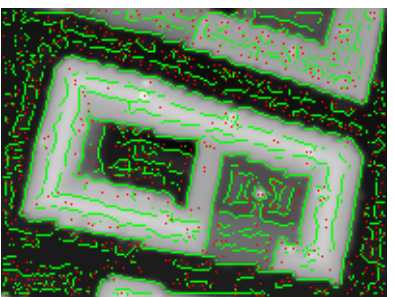

(j) Harris

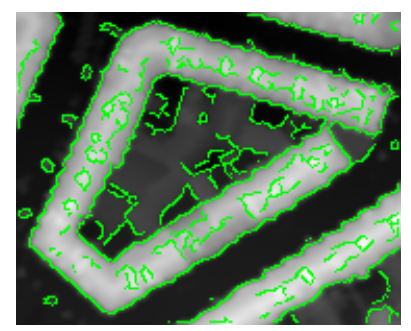

(c) Canny

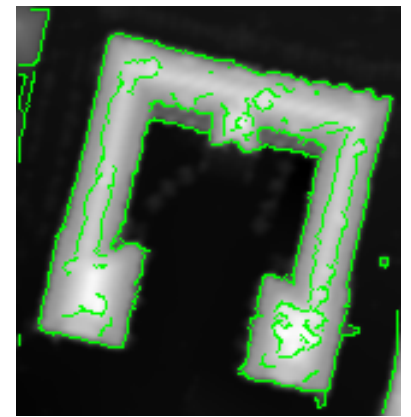

(g) Canny

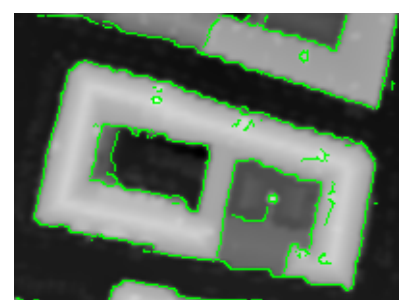

(k) Canny

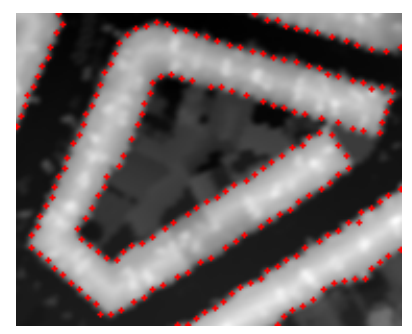

(d) SPP

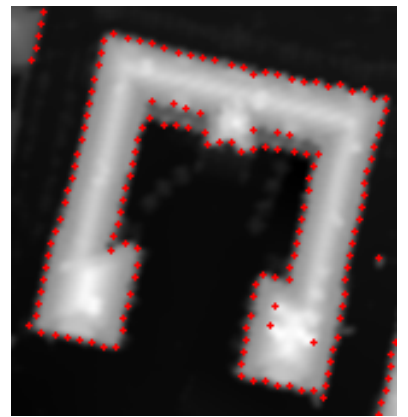

(h) SPP

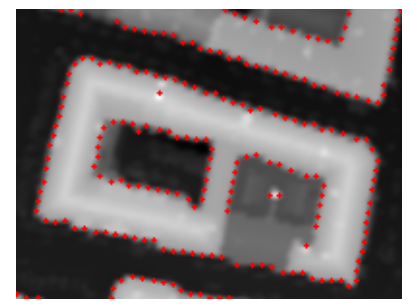

(1) SPP

Figure 6: Experimental results: The images and features detected by the Harris, the Canny and the new spatial point process based detector are indicated by the captions. The corners and edges are depicted as red and green pixels, respectively. The points on the linear features are also depicted as red crosses. 


\section{REFERENCES}

Alahi, A., Ortiz, R. and Vandergheynst, P., 2012. Freak: Fast retina keypoint. In: 2012 IEEE Conference on Computer Vision and Pattern Recognition (CVPR), pp. 510-517.

Arbelaez, P., Maire, M., Fowlkes, C. and Malik, J., 2011. Contour detection and hierarchical image segmentation. IEEE Transactions on Pattern Analysis and Machine Intelligence 33(5), pp. 898-916.

Baddeley, A. J. and Lieshout, M. V., 1993. Stochastic geometry models in high-level vision. Journal of Applied Statistics 20(5-6), pp. 231-256.

Bay, H., Ess, A., Tuytelaars, T. and Van Gool, L., 2008. Speededup robust features (surf). Computer Vision and Image Understanding 110(3), pp. 346-359. Similarity Matching in Computer Vision and Multimedia.

Canny, J., 1986. A computational approach to edge detection. IEEE Transactions on Pattern Analysis and Machine Intelligence (6), pp. 679-698.

Chai, D., Förstner, W. and Lafarge, F., 2013. Recovering linenetworks in images by junction-point processes. In: IEEE Conference on Computer Vision and Pattern Recognition (CVPR), pp. 1894-1901.

Deriche, R., 1987. Canny's criteria to derive a recursively implemented optimal edge detector. International Journal of Computer Vision 1, pp. 167-187.

Dubrovina, A., Rosman, G. and Kimmel, R., 2015. Multi-region active contours with a single level set function. IEEE Transactions on Pattern Analysis and Machine Intelligence 37, pp. 15851601.

Förstner, W. and Gülch, E., 1987. A fast operator for detection and precise location of distinct points, corners and centres of circular features. ISPRS Intercommission Conference on Fast Processing of Photogrammetric Data pp. 281-305.

Green, P. J., 1995. Reversible jump markov chain monte carlo computation and bayesian model determination. Biometrika 82(4), pp. 711-732.

Harris, C. and Stephens, M., 1988. A combined corner and edge detector. In: Alvey Vision Conference, Vol. 15, Citeseer, pp. 147151 .

Kadir, T., Zisserman, A. and Brady, M., 2004. An affine invariant salient region detector. In: Computer Vision-ECCV 2004, Springer, pp. 228-241.

Kass, M., Witkin, A. and Terzopoulos, D., 1988. Snakes: Active contour models. International Journal of Computer Vision 1(4), pp. 321-331.

Kirkpatrick, S., Gelatt, C. D. and Vecchi, M. P., 1983. Optimization by simulated annealing. Science 220(4598), pp. 671-680.

Lacoste, C., Descombes, X. and Zerubia, J., 2005. Point process for unsupervised line network extraction in remote sensing. IEEE Transactions on Pattern Analysis and Machine Intelligence 27(10), pp. $1568-1579$.

Lacoste, C., Descombes, X. and Zerubia, J., 2010. Unsupervised line network extraction in remote sensing using a polyline process. Pattern Recognition 43(4), pp. 1631-1641.

Lafarge, F., Gimelarb, G. and Descombes, X., 2010. Geometric feature extraction by a multi-marked point process. IEEE Transactions on Pattern Analysis and Machine Intelligence 32(9), pp. 1597-1609.
Leutenegger, S., Chli, M. and Siegwart, R. Y., 2011. Brisk: Binary robust invariant scalable keypoints. In: 2011 IEEE International Conference on Computer Vision (ICCV), pp. 2548-2555.

Lowe, D., 2004. Distinctive image features from scale-invariant keypoints. International Journal of Computer Vision 60(2), pp. 91-110.

Martin, D. R., Fowlkes, C. C. and Malik, J., 2004. Learning to detect natural image boundaries using local brightness, color, and texture cues. IEEE Transactions on Pattern Analysis and Machine Intelligence 26(5), pp. 530-549.

McIlhagga, W., 2011. The canny edge detector revisited. International Journal of Computer Vision 91(3), pp. 251-261.

Ming, Y., Li, H. and He, X., 2013. Winding number for region-boundary consistent salient contour extraction. In: 2013 IEEE Conference on Computer Vision and Pattern Recognition (CVPR), pp. 2818-2825.

Mishra, A. K., Fieguth, P. W. and Clausi, D. A., 2011. Decoupled active contour (dac) for boundary detection. IEEE Transactions on Pattern Analysis and Machine Intelligence 33(2), pp. 310 324.

Møller, J. and Waagepetersen, R., 2004. Statistical inference and simulation for spatial point processes. Chapman \& Hall/CRC.

Ortner, M., Descombes, X. and Zerubia, J., 2007. Building outline extraction from digital elevation models using marked point processes. International Journal of Computer Vision 72(2), pp. 107-132.

Ortner, M., Descombes, X. and Zerubia, J., 2008. A marked point process of rectangles and segments for automatic analysis of digital elevation models. IEEE Transaction on Pattern Analysis and Machine Intelligence 30(1), pp. 105-119.

Payet, N. and Todorovic, S., 2013. Sledge: sequential labeling of image edges for boundary detection. International Journal of Computer Vision 104(1), pp. 15-37.

Perrin, G., Descombes, X. and Zerubia, J., 2005. A marked point process model for tree crown extraction in plantations. In: Proc. of IEEE International Conference on Image Processing, pp. 1-4.

Ren, X., 2008. Multi-scale improves boundary detection in natural images. In: Computer Vision-ECCV 2008, Springer, pp. 533545.

Rosten, E. and Drummond, T., 2006. Machine learning for highspeed corner detection. In: A. Leonardis, H. Bischof and A. Pinz (eds), Computer Vision - ECCV 2006, Lecture Notes in Computer Science, Vol. 3951, Springer, pp. 430-443.

Rublee, E., Rabaud, V., Konolige, K. and Bradski, G., 2011. Orb: an efficient alternative to sift or surf. In: 2011 IEEE International Conference on Computer Vision (ICCV), pp. 2564-2571.

Schmidt, A., Rottensteiner, F., Soergel, U. and Heipke, C., 2015. A graph based model for the detection of tidal channels using marked point processes. The International Archives of the Photogrammetry, Remote Sensing and Spatial Information Sciences XL-3/W3, pp. 115-121.

Shi, J. and Tomasi, C., 1994. Good features to track. In: IEEE Computer Society Conference on Computer Vision and Pattern Recognition, IEEE, pp. 593-600.

Smith, S. and Brady, J., 1997. Susan - a new approach to low level image processing. International Journal of Computer Vision 23(1), pp. 45-78

Steger, C., 1998. An unbiased detector of curvilinear structures. IEEE Transactions on Pattern Analysis and Machine Intelligence 20(2), pp. 113-125. 
Stoica, R., Descombes, X. and Zerubia, J., 2004. A gibbs point process for road extraction from remotely sensed images. International Journal of Computer Vision 37(2), pp. 121-136.

Tomasi, C. and Kanade, T., 1991. Detection and tracking of point features. School of Computer Science, Carnegie Mellon Univ. Pittsburgh.

Tu, Z. and Zhu, S.-C., 2002. Image segmentation by data-driven markov chain monte carlo. IEEE Transactions on Pattern Analysis and Machine Intelligence 24(5), pp. 657-673.

Tuytelaars, T. and Van Gool, L., 2004. Matching widely separated views based on affine invariant regions. International Journal of Computer Vision 59(1), pp. 61-85.

Xu, Q., Varadarajan, S., Chakrabarti, C. and Karam, L. J., 2014. A distributed canny edge detector: Algorithm and fpga implementation. IEEE Transactions on Image Processing 23(7), pp. 2944-2960.

Zhu, Q., Song, G. and Shi, J., 2007. Untangling cycles for contour grouping. In: IEEE 11th International Conference on Computer Vision, pp. 1-8. 